\title{
WIRTSCHAFTLICHKEITSPROGNOSEN FÜR AUTOMATENSTANDORTE
}

\author{
Chantal Wentler, Tilo Wendler, Bertil Haack
}

\section{Zusammenfassung}

Die Leistung und Wirtschaftlichkeit eines Automaten sind nicht nur für ein großes Unternehmen wie die Deutsche Bahn AG zwei wichtige Kriterien bei der Suche nach einem guten Standort für ihre Ticketautomaten. Mindestens ebenso wichtig sind die Akzeptanz und Zufriedenheit der Kunden, denn deren Bedürfnisse sind wichtige Indikatoren für die Betreiber der Automaten. Im vorliegenden Beitrag sollen die Grundzüge eines Prognosemodells für die wirtschaftliche Bewertung von Automatenstandorten vorgestellt werden (Kapitel 2). Das Prognosemodell wird auf dessen Anwendbarkeit an konkreten Einzelfällen geprüft (Kapitel 3) und daraus werden anschließend Anforderungen an ein verfeinertes Prognosewerkzeug abgeleitet (Kapitel 4).

\begin{abstract}
The performance and economic efficiency of a machine are not only for a big enterprise like Deutsche Bahn AG two important criteria with the search for a good location for a ticket machines. The acceptance and satisfaction of the customers is at least as important, because their requirements are important indicators for the operators of the machine. In the present article the main features of a forecast model should be introduced for the economic assessment by machine locations (chapter 2). The forecast model is checked for his applicability in concrete isolated cases afterwards (chapters 3 ) and from it demands for refined forecast tools are derived (chapter 4 ).
\end{abstract}

\section{»I. EINLEITUNG:}

Ein Blick in Einkaufszentren, Flughäfen, Bahnhöfe oder Geschäftsstraßen zeigt, dass unser tägliches Leben durch eine Vielzahl von Automaten geprägt ist. Wir können mit ihrer Hilfe Getränke, Zigaretten, Süßigkeiten und andere Waren des täglichen Bedarfs kaufen, Streckenauskünfte erhalten und Fahrkarten erwerben, Geld abheben und einzahlen, Kontostände anzeigen lassen und Daueraufträge einrichten und vieles mehr.

Automaten sind nicht aus unserem Leben wegzudenken und haben etwas derart Selbstverständliches an sich, dass wir uns eher selten tiefergehende Gedanken über sie machen: Wie wird eigentlich der im Idealfall reibungsfreie Betrieb der Automaten sichergestellt? Wie viel kostet der Betrieb den Anbieter? Warum gibt es genau an dieser Stelle einen Geldautomaten oder einen Kontoauszugsdrucker? Warum hat der Automat genau diese Funktionalität etwa bargeldloser und bargeldhafter Verkauf von Bahntickets - und keine weitere? Wie kann die Sicherheit von Automaten beispielsweise gegen Einbruch und Diebstahl gewährleistet werden?

Unabhängig davon, wie die Antworten im Einzelfall lauten, ist es naheliegend, dass sie überaus eng mit der
Frage nach der Wirtschaftlichkeit des jeweiligen Automaten verknüpft sind. Von strategisch geprägten Ausnahmen abgesehen - z. B. „Wir müssen an diesem Standort einen Geldautomaten aufstellen, weil auch die Bank A als unser Wettbewerber dort mit einem Automaten vertreten ist. " - muss der Automat rentabel sein. D. h., die mit dem Betrieb des Automaten verbundenen Einnahmen müssen die entsprechenden Ausgaben übertreffen.

Wie kann ein derartiger Automatenstandort gefunden werden, ohne dass zu sehr auf „Versuch und Irrtum“ abgestellt wird und die damit häufig verbundenen Risiken (sprich: erhebliche Kosten) die Oberhand gewinnen?

Die Suche nach Automatenstandorten wird derzeit im Wesentlichen mittels dreier Verfahren betrieben:

\section{Erfahrungsgestützte Entscheidungen von Experten}

Auf Basis jahrelanger Erfahrung ist es möglich, die Qualität von Standorten zu bewerten. Dabei werden verschiedene Kriterien mehr oder weniger unbewusst und unstrukturiert in die Überlegung einbezogen.

\section{Computergestützte Bewertung}

Automatenhersteller bieten interessierten Kunden in der Regel umfangreiche
Softwarewerkzeuge zur Bewertung potenzieller Standorte an. Die Systeme sind sehr komplex und beruhen meist auf umfangreichen Annahmen zur Anzahl von Ein- und Auszahlungsvorgängen.

\section{Geschäftspolitisch vorgeprägte Entscheidungen}

Aufgrund spezifischer Wettbewerbskonstellationen vor Ort sind beispielsweise Kreditinstitute gezwungen, an bestimmten Orten Automaten zu installieren. Diese Entscheidungen beruhen in der Regel nicht auf betriebswirtschaftlichen Überlegungen, die den jeweiligen konkreten Automaten betreffen. Oftmals dienen sie der Abschottung des eigenen lokalen Marktes. Das kann so weit gehen, dass Kreditinstitute aus geschäftspolitischer Sicht und im Zuge politischer Einflüsse - wie sie zum Beispiel aus dem Bereich der Öffentlichen Banken oder der Genossenschaftsbanken bekannt sind - gezwungen sind, an unrentablen Orten Automaten $\mathrm{zu}$ installieren. Oft trifft dies Standorte, an denen sie aufgrund der Schließung von Filialen keinen persönlichen Service mehr anbieten.

Während der dritte und letzte Fall keinem betriebswirtschaftlichen Argument zugänglich ist, können die ersten beiden Ansätze als Maßstab für eine 
neu zu entwickelnde Methodik genutzt werden.

Fall 2, die computergestützte Bewertung einzelner Standorte, ist arbeitsund kostenintensiv. Entscheidungen auf Basis von Expertenwissen können genutzt werden, um ein alternatives Vorgehensmodell zu entwerfen (vgl. Moro 2004: 157).

Dieses ist insbesondere dann von Interesse, wenn der zu installierende Automat neue, bisher nicht verfügbare oder verbreitete Funktionalitäten anbietet - z. B. Verkauf von Kinokarten am ÖPNV-Fahrkartenautomaten. Ziel ist es, vor der Aufstellung eines Automaten mit einer gewissen Sicherheit (Wahrscheinlichkeit) prognostizieren zu können, ob es wirtschaftlich lohnenswert ist, die geplanten Selbstbedienungsfunktionen am vorgesehenen Standort anzubieten (vgl. Becker et al. 2010: 144 ff.).

\section{» II. IDENTIFIKATION WIRTSCHAFT- LICHER AUTOMATENSTANDORTE}

\section{Grundüberlegungen}

Ausgangssituation für die Entwicklung des nachfolgend beschriebenen Prognosemodells war die Zusammenarbeit der Autoren mit Mitarbeitern der Kooperationspartner SHC Stolle und Heinz Consultants (SHC) und Deutsche Bahn Kommunikationstechnik (DB KT) der TH Wildau hinsichtlich der Optimierung von Geldkreisläufen auf Bahnhöfen.

Dabei standen die Ticketautomaten der Deutschen Bahn (DB) im Zentrum der Überlegungen: Während Techniker die Automaten durchschnittlich alle 30 Tage warten, müssen stark frequentierte Geräte zudem alle ein bis zwei Tage von einem Werttransportunternehmen (WTU) bedient werden, um das durch die Ticketverkäufe vereinnahmte und in einer Kassette im Automaten gesammelte Bargeld zu entnehmen und sicher zu einer Bank zu transportieren.

Im Sinne der Wirtschaftlichkeit der Automaten wäre es von erheblichem Vorteil, insbesondere die Anzahl der kostenintensiven WTU-Fahrten deutlich zu reduzieren. Dazu ist eine geeignete Steuerung der Bargeldmenge im
Automaten erforderlich. Im Idealfall sollte sich diese in einer Art Gleichgewichtszustand bzw. auf gleichbleibendem Niveau befinden. Sowohl zu viel Bargeld aus Einzahlvorgängen als auch fehlendes Bargeld als Wechselgeld führen zur automatischen Außerbetriebnahme des Automaten. Ideal wäre es, wenn sich Einzahlungen und Auszahlungen im Mittel die Waage hielten, so dass der Bargeldbestand im Automaten im Durchschnitt einem Betrag $X$ entspräche, der deutlich unter dem Bestand für „zu viel Bargeld“ und klar über der Geldmenge für „zu wenig Bargeld“ läge.

Derzeit kann ein derartiges Gleichgewicht der Automaten nicht erreicht werden, da in der Regel immer mehr Bargeld durch Ticketverkäufe eingenommen als in Form von Wechselgeld wieder herausgegeben wird (Statista 2012).

Das Geldniveau im Automaten kann deutlich länger im betriebsfähigen Zustand gehalten werden, indem die Ticketautomaten durch eine so genannte Cash Recycling-Funktion (vgl. etwa http://www.stolleundheinz.com) ergänzt werden. Hierbei wird das jeweilige Gerät um eine technische Komponente erweitert, die es Ticketkäufern - ähnlich wie im Supermarkt REWE - ermöglicht, Bargeld bis zu einer Höhe von EUR 200,00 kostenfrei auszuzahlen (Rönisch 2008: 14).

Abgesehen von der Klärung rechtlicher Fragen, die hier nicht betrachtet werden (Wentler 2011), erfordert die Ausstattung eines Ticketautomaten mit der beschriebenen Cash RecyclingFunktion nach Auskunft von DB-Spezialisten ein Investment von etwa EUR 8.000. Solche Investitionen sind zuvor detailliert zu prüfen. Denn bereits ein gedanklicher Vergleich der Automatenstandorte Hauptbahnhof Berlin und Bahnhof Königs Wusterhausen lässt vermuten, dass sich diese Ausgaben nicht an jedem Standort amortisieren wird.

Dies führt zu dem eingangs adressierten Thema des Bewertungsmodells am Beispiel der Ticketautomaten. Die zu klärende Frage „Für welche Ticketautomaten erscheint die Erweiterung um eine Cash Recycling-Funktionalität wirtschaftlich sinnvoll?" muss bundesweit untersucht werden. Da sich die bisherigen Verfahren zur Bewertung von Automatenstandorten derartigen Szenarien verschließen, ist die Entwicklung neuer Ansätze erforderlich.

Antworten müssen in die Richtung zielen, das bekannte Investment in den jeweiligen Automaten (s. o.) den zu erwartenden Kosteneinsparungen aufgrund der möglichen Reduktion von WTU-Fahrten sowie den denkbaren Mehreinnahmen aufgrund der insgesamt längeren Verfügbarkeit des Automaten durch längerfristige Verhinderung des Zustandes „Außer Betrieb“ aufgrund von zu wenig oder zu viel Bargeld gegenüber zu stellen.

Mit Blick auf die eingangs formulierten Überlegungen bedeutet das, die Wahrscheinlichkeit von Kosteneinsparungen und etwaigen Mehreinnahmen je Ticketautomat zu prognostizieren. Anders formuliert geht es darum, vorherzusagen, wie wahrscheinlich es ist, dass die besagte Automatenfunktionalität an einem Automatenstandort von den Kunden angenommen wird.

\section{Zwei Schritte zur Prognose}

Die Prognose wird hier zunächst in generalisierter Form betrachtet. Grundlage ist ein beliebiger Automat mit einer beliebigen Selbstbedienungsfunktionalität. Dann sind folgende zwei Aspekte aus qualitativer Sicht vorrangig:

1. Rahmenbedingungen, Vorgaben, Fakten o. ä., die eine Vorentscheidung für oder gegen den Automateneinsatz ermöglichen und

2. die detaillierte Feststellung der Eignung vorausgewählter Umgebungen für den jeweiligen Automatenbetrieb.

Der erste Schritt gestattet, nicht-profitabel erscheinende Standorte relativ schnell und mit wenig Aufwand auszuschließen. Der zweite, aufwändigere Schritt der Detailprüfung wird damit nur für aussichtsreiche Standorte durchgeführt.

Wichtig ist, dass sich das Prognosemodell als valide und reliabel erweist. Es muss tatsächlich Prognosen für Wirtschaftlichkeit der betrachteten Automaten liefern und diese müssen möglichst gut reproduzierbar und damit 
möglichst weitgehend unabhängig von subjektiven Einschätzungen sein.

Diesen Forderungen wird zunächst dadurch Rechnung getragen, dass der erste Schritt auf Regeln aufsetzt, die vor Beginn der Prognose mit Fachleuten festgelegt werden. Im zweiten Schritt wird dann darauf geachtet, dass die notenmäßige Bewertung eines Automatenstandortes einerseits anhand möglichst formaler Kriterien erfolgt und dass andererseits die jeweiligen Noten eindeutig auf Basis nachprüfbarer Ausprägungen der Kriterien vergeben werden. Diese Kriterien wie beispielsweise „Entfernung des Automaten vom nächstgelegenen Geldautomaten" und die Benotungen wie etwa "sehr gut" bei „Entfernung von mehr als 200 m" sind bereits im Modell mit Fachleuten festgelegt worden.

Auf Basis des Modells sind diejenigen Bahnhöfe zu identifizieren, die für einen rentablen Betrieb sprechen. Nach der Identifikation der entsprechenden Bahnhöfe erfolgt die Betrachtung der auf den Bahnhöfen bereits installierten Ticketautomaten. Wird in der ersten Untersuchung zum Beispiel München Hauptbahnhof als Bahnhof mit einem rentablen Betrieb identifiziert, so muss anschließend entschieden werden, welche der dortigen Ticketautomaten für eine potenzielle Umrüstung in Frage kommen. Hierzu wurden u. a. die nachfolgenden DB-spezifischen Informationen je Ticketautomat zu Rate gezogen:

Qualität der Verkehrsanbindung
des Standortes
- Pendelverkehr zu einem Flughafen
- Geschäfte für täglichen Bedarf,
Dienstleistungen
- ...
Eignung der Umgebung für den
Automatenbetrieb
- Standort des Automaten DIREKT IM
Kundenstrom
- Entfernung des zu bewertenden
Automaten von einem Geldautomaten
(FremdGAA) einer anderen bekannten
Institutsgruppe.
- ...

Anzahl der Ticketverkäufe gesamt,

Verhältnis der bar- und unbaren Zahlungen,

Servicekosten für das Werttransportunternehmen sowie

sonstige Kosten für den Ticketautomaten.

\section{Diskussion der Modell bestand teile}

Grundsatz der Modellerstellung war, dass jedes der Kriterien objektiv auf ein und derselben Skala bewertet werden kann. Einige der im Modell eingesetzten Kriterien sind aus Tabelle 1 ersichtlich. In der Modellvalidierung hat sich bestätigt, dass eine Vorgabe der Bewertungskriterien nicht davon befreit, die Skalenwerte (Noten) selbst dezidiert vorzugeben. Aufgrund der sonst vorhandenen subjektiven Bewertungsmaßstäbe würden unterschiedliche Gutachter bei gleichen Standorten zu unterschiedlichen Resultaten gelangen (Subjektivität). Am Beispiel des schwer zu evaluierenden Kriteriums „Standort des Automaten DIREKT IM Kundenstrom" sei der Ansatz verdeutlicht. Die hier zugrunde gelegten Skalenwerte (Ausprägungen des Kriteriums) sind:

1. Automat ist sehr gut sichtbar, steht jedoch nicht direkt im Kundenstrom,

2. Automat steht ETWAS abseits vom Kundenstrom, Kunde könnte sich jedoch zeitweilig bedrängt fühlen,

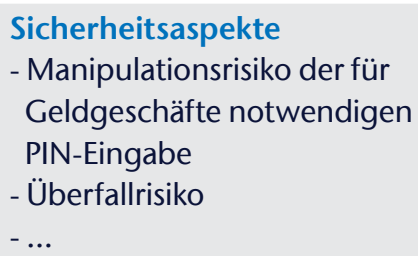

Objekteigenschaften (Hardfacts)

- Möglichkeit der direkten Kameraüberwachung des Automatenstandortes

- Beleuchtung/Sicherheitsgefühl

- Zugang zu Fahrstuhl oder Rolltreppe ...
3. Automat steht direkt im Kundenstrom, Kunde fühlt sich oft bedrängt. Die an einem Automatenstandort zutreffende Ausprägung des Kriteriums lässt sich unabhängig von der Person, die die Bewertung des Standortes vornimmt, ermitteln.

Mit einer Vielzahl derartiger Kriterien soll die Wahrscheinlichkeit abgeschätzt werden, dass der Kunde am betreffenden Automaten eine Transaktion mit Bargeldtransfer durchführt.

\section{»III. ANWENDUNG DES MODELLS}

Mit dem Bewertungsmodell wurden bisher Ticketautomaten an verschiedenen Bahnhöfen untersucht. Darunter u. a.:

\section{Berlin Hauptbahnhof}

Berlin Alexanderplatz

\section{Berlin Gesundbrunnen}

Berlin Südkreuz

Königs Wusterhausen

Beispielhaft sei die Analyse des Bahnhofs Alexanderplatz betrachtet, bei der u. a. diejenigen Automaten analysiert wurden, die direkt vor dem Reisezentrum der Deutschen Bahn AG aufgestellt sind (am Bahnhof Berlin Alexanderplatz befindet sich zurzeit nur ein Reisezentrum der Deutschen Bahn AG; neben dessen Eingang stehen insgesamt vier Ticketautomaten; hiervon wurde einer ausgewählt).

Die Analyse des oben abgebildeten Automaten mittels unseres Bewertungsmodells hat hinsichtlich des Kriteriums Qualität der Verkehrsanbindung sowohl in der Kategorie ÖPNV als auch in der Kategorie Pendelverkehr zu einem Flughafen eine positive Einschätzung geliefert. Konkret liegen beim ÖPNV vier Anbindungsmöglichkeiten an S-Bahnlinien und drei Anbindungsmöglichkeiten an U-Bahnlinien vor (Deutsche Bahn 2012). Diese stellen unter anderem sicher, dass der Kunde vom Bahnhof Alexanderplatz jeden der beiden jetzigen Berliner Flughäfen 


\section{Ebenen}

1223

\begin{tabular}{l|r}
1 & 2 \\
\hline & 2
\end{tabular} 2 3

\section{Standort}

Name der Lokation des Automaten

Nummer des Fahrkartenautomaten

Gebäude/Etage

Verbale Beschreibung des Standortes

(z. B. Eingangshalle links, ...)

Herstellername:

Typenbezeichnung:

\section{Qualitative Bewertungsaspekte}

\begin{tabular}{|c|c|c|c|c|}
\hline 1 & \multicolumn{4}{|c|}{ Qualität der Verkehrsanbindung des Standortes } \\
\hline 1 & ÖPNV & $\begin{array}{l}\text { </=3 Anbindungsmög- } \\
\text { lichkeiten }\end{array}$ & $\begin{array}{l}2 \text { Anbindungsmöglich- } \\
\text { keiten }\end{array}$ & $\begin{array}{l}\text { mind. eine Anbin- } \\
\text { dungsmöglicheit }\end{array}$ \\
\hline 2 & $\begin{array}{l}\text { Pendelverkehr zu } \\
\text { einem Flughafen }\end{array}$ & $\begin{array}{l}\text { Es besteht direkter } \\
\text { Anschluss an Pendelver- } \\
\text { kehr, mind. } 1 \text { Möglich- } \\
\text { keit (Bus, S-Bahn) }\end{array}$ & $\begin{array}{l}\text { Indirekte Verbindung, } \\
1 \times \text { umsteigen }\end{array}$ & $\begin{array}{l}\text { Indirekte Verbindung, } \\
\text { ab 2x umsteigen }\end{array}$ \\
\hline 3 & $\begin{array}{l}\text { Geschäfte für täglichen } \\
\text { Bedarf, Dienstleistun- } \\
\text { gen }\end{array}$ & $\begin{array}{l}\text { AT ist gut sichtbar bzw. } \\
\text { gut ausgeschildert }\end{array}$ & $\begin{array}{l}\text { AT steht etwas abseits, } \\
\text { jedoch noch ausge- } \\
\text { schildert }\end{array}$ & $\begin{array}{l}\text { AT steht abseits und ist } \\
\text { nicht ausgeschildert }\end{array}$ \\
\hline
\end{tabular}

Abb. 1) Teilausschnitt aus dem entwickelten Bewertungsmodell (eigene Abbildung)

Tegel und Schönefeld durch regelmäßigen Pendelverkehr erreichen kann.

Innerhalb des Bahnhofes hat der Kunde nicht nur die Möglichkeit, sich ein Ticket zu kaufen, sondern auch, Einkäufe zu tätigen. Diese Möglichkeit kann die Entscheidung unterstützen, ob die Integration einer Cash Recycling-Funktion in den Ticketautomaten erfolgen soll.

Bei der weiteren Bewertung des Ticketautomaten sind die Sicherheitsaspekte nicht zu unterschätzen. Dieses Kriterium wird im vorliegenden Modell als Überfallrisiko und Manipulationsrisiko der für Geldgeschäfte notwendigen PIN-Eingabe bezeichnet. Die Untersuchung des Automaten hat gezeigt, dass das Überfallrisiko von Ticketautomaten oft als gering einzuschätzen ist. Eine bereits vorhandene Kennzeichnung am Automaten, die auf die versteckte Kameraüberwachung am Standort hinweist, schränkt die Möglichkeit eines potenziellen Überfalls zusätzlich ein. Des Weiteren deckt ein angrenzendes Reisezentrum mit den Öffnungszeiten den größten Teil der Tageszeit ab und

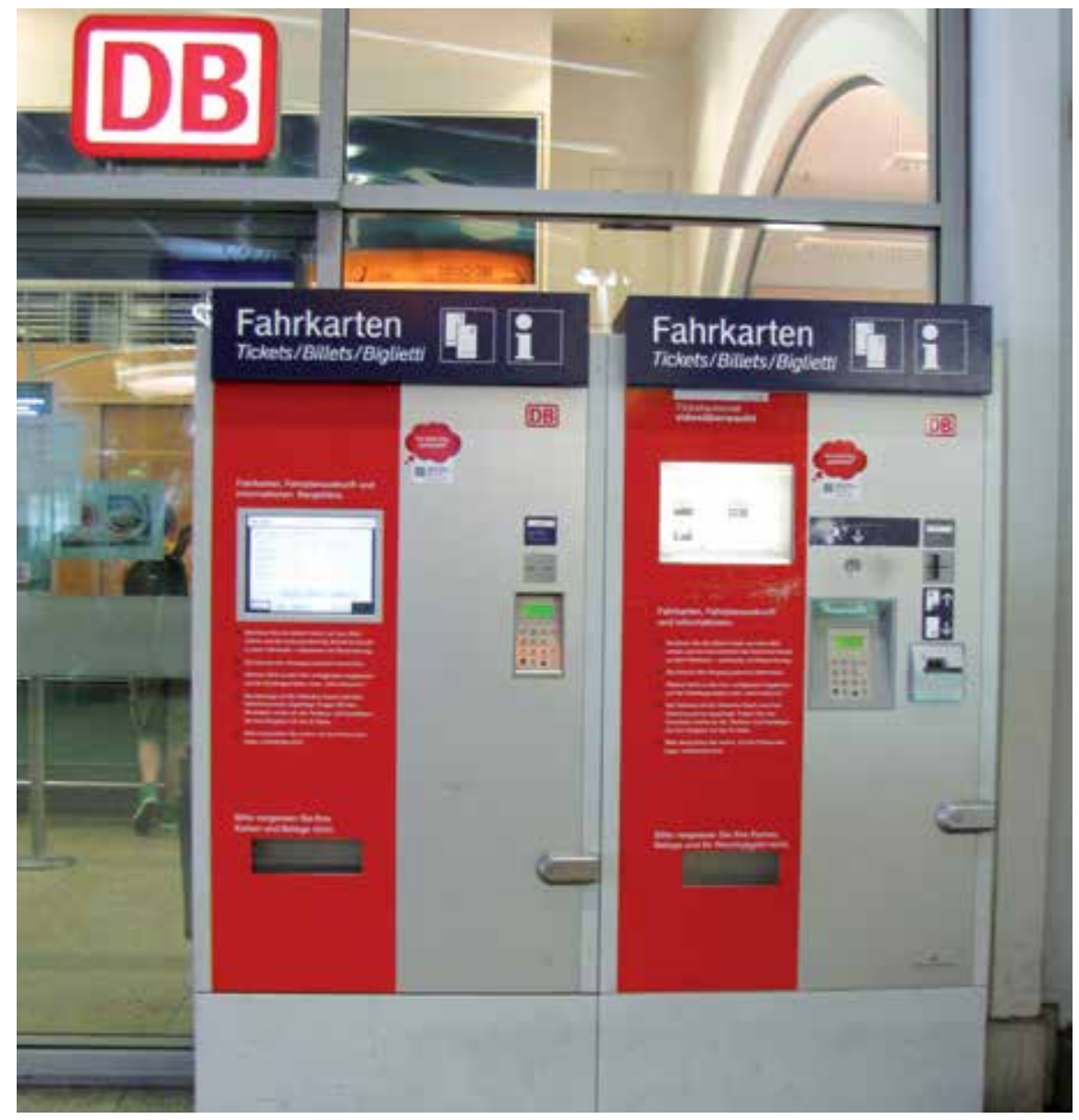

Abb. 2) Ticketautomat Berlin-Alexanderplatz 
verringert die Gefahr eines Überfalls während der Öffnungszeiten.

Das Manipulationsrisiko an dem für die Geldgeschäfte notwendigen PIN-Pad kann nie vollständig ausgeschlossen werden. Bei den untersuchten Ticketautomaten kann das Anbringen von fremden Zusatzbauteilen am Kartenschlitz oder am PIN-Pad sowie von Zusatzkameras zur Überwachung des Eingabefeldes aufgrund der Bauart des Automaten nicht ausgeschlossen werden. Befindet sich der Kunde direkt vor dem Ticketautomaten und wird aufgefordert seine PIN über das PIN-Pad einzugeben, verdeckt er mit seinem Körper den Blick auf das PIN-Pad nicht vollständig. Die Einstufung des Manipulationsrisikos erfolgt auf mittlerer Stufe.

Das Bewertungsmodell gibt abschließend Auskunft über die Objekteigenschaften, die so genannten Hardfacts. Hierunter fallen zum Beispiel die Architektur und die Bauweise des Bahnhofs. Bei der Untersuchung am Bahnhof Alexanderplatz ergibt sich für das Kriterium Beleuchtung/Sicherheitsgefühl eine Einteilung im mittleren Bereich. Der Ticketautomat wird nicht vollständig beleuchtet und erfüllt die Anforderung der Beleuchtung auf Tageslichtniveau nicht vollständig.

Mit den durchgeführten Untersuchungen und dem validierten Modell wird eine Entscheidungshilfe für die Ausrüstung von Ticketautomaten mit erweiterten Funktionen geboten. Ein untersuchter Automat kann anhand aller empirisch gewonnenen Daten als Kandidat für eine mögliche Umrüstung sehr gut identifiziert oder ausgeschlossen werden.

\section{»IV. ANFORDERUNGEN AN EIN VERFEI- NERTES PROGNOSEWERKZEUG}

Die Anwendung des dargestellten Bewertungsmodells erlaubt es, subjektive Einschätzungen durch objektive Bewertungen zu ersetzen und gibt so deutlich mehr Sicherheit bei der Bewertung von Automatenstandorten als die eingangs erwähnten derzeit üblichen drei Methoden.

Allerdings ist es notwendig, das Modell um einen Schritt zu erweitern, um auch quantitative Aspekte in der Untersuchung zu berücksichtigen: Die ausschließliche Betrachtung qualitativer Aspekte kann zu Fehleinschätzungen führen. Um die Frage, ob ein Automat rentabel ist und damit am Ende höhere Einnahmen als Ausgaben entstanden sind, möglichst genau beantworten zu können, müssen die Wahrscheinlichkeitsannahmen zusätzlich um eine Kostenbetrachtung ergänzt werden.

Anhand eines Beispiels soll dieser noch notwendige dritte Schritt verdeutlicht werden. Betrachtet wird ein DB-Ticketautomat, der am Bahnhof in GarmischPartenkirchen aufgestellt ist. Dieser Automat weist „passable“ Ticketverkäufe aus, so dass er durchschnittlich nur einmal pro Woche von einem WTU angefahren werden muss. Im Vergleich mit den Automaten auf dem Berliner Hauptbahnhof, die alle ein bis zwei Tage von einem WTU geleert werden müssen, könnte die Einschätzung „keine Cash Recycling-Funktion erforderlich" lauten. Tatsächlich kommt aber das beauftragte Werttransportunternehmen für die Leerung des Ticketautomaten aus München und fährt nach Beendigung der Leerung wieder dorthin zurück.

Eine Hochrechnung inkl. der Fahrtkosten für etwa $90 \mathrm{~km}$ pro Richtung zeigt auf quantitativer Basis eine sinnvolle Umrechnung an (Google Maps 2012).

Die Attraktivität einer Cash Recycling-Funktion am Standort Garmisch Partenkirchen ist damit deutlich gestiegen. Sie wächst außerdem, wenn zusätzlich die WTU-Gebühr für die Dienstleistung in Betracht gezogen und berücksichtigt wird, dass die Ausrüstung eines Fahrscheinautomaten mit Cash Recycling-Funktionalität laut Experten der DB eine hardwareseitige Investition von ca. EUR 8.000 erfordert.
Mit dem dreistufigen Modell ist es sehr gut möglich, diejenigen Ticketautomaten für eine Umstellung auf Cash Recycling zu identifizieren, deren Amortisation wahrscheinlich ist.

Die eingehende Fragestellung „Ist die Integration einer Cash Recycling-Funktion in den Ticketautomaten rentabel für die Deutsche Bahn AG?" kann in Zukunft auch in Zusammenhang mit anderen möglichen Interessenten gestellt werden. Das dreistufige Modell kann generell nicht nur bei der Entscheidung über die Umrüstung von Ticketautomaten helfen, sondern zum Beispiel auch für die Umrüstung von Geldautomaten oder Warenautomaten genutzt werden. 


\section{LITERATURVERZEICHNIS}

Becker, J. et al. (2010): Preisfindung für hybride Leistungsbündel - Modellbasierte Integration von Ansätzen zur

Entscheidungsunterstützung aus „Dienstleistungsmodellierung 2010“, 144-166, Springer.

Deggendorfer Forum zur digitalen Datenanalyse e. V. (2009): Digitale Datenanalyse, Interne Revision und Wirtschaftsprüfung: Synergien nutzen - Prüfungen optimieren, Erich Schmidt Verlag.

Deutsche Bahn AG (2012):

http://www.bahn.de/p/view/mdb/pv/planen_buchen/ liniennetzkarten/2011/MDB85587-liniennetz_bb_2011. pdf, Zugriff am 04.09.2012.

Google Maps (2012):

http://maps.google.de/maps?hl=en\&tab=wl, Zugriff am 04.09.2012.

Moro, M. (2004): Modellbasierte Qualitätsbewertung von Softwaresystemen: Bewertung von Softwarearchitekturen in Bezug auf ihren Erfüllungsgrad der Qualitätsanforderungen, Books on Demand $\mathrm{GmbH}$.

Rönisch, P. (2008): Direktbanken in Deutschland - Stand und Perspektiven, GRIN Verlag.

Stolle und Heinz Consulting 2012: http://www.stolleundheinz.com/, Zugriff am 05.09.2012.

Statista (2012): http://de.statista.com/themen/747/ bargeldlose-zahlungen/, Zugriff am 05.09.2012.

Wentler, C. (2011): Belegarbeit im Studienfach Empirische Methoden mit dem Titel: „Bewertungsmodell für Evaluation eines Ticketautomaten zur Umrüstung auf Cash Recycling bei der Deutschen Bahn AG“, TH Wildau.

\section{ABBILDUNGSVERZEICHNIS}

Abbildung 1:

Teilausschnitt aus dem entwickelten Bewertungsmodel

Abbildung 2:

Ticketautomat Berlin-Alexanderplatz

\section{AUTOREN}

Chantal Wentler

Studierende im Bachelor-Studiengang

"Europäisches Management"

Fachbereich Wirtschaft, Verwaltung und Recht

TH Wildau [FH]

chantal.wentler@th-wildau.de

Prof. Dr. Tilo Wendler

Fachbereich Wirtschaft, Verwaltung und Recht

TH Wildau [FH]

tilo.wendler@th-wildau.de

\section{Prof. Dr. Bertil Haack}

Dekan

Fachbereich Wirtschaft, Verwaltung und Recht

TH Wildau [FH]

bertil.haack@tfh-wildau.de 